 \\ International Journal of Autism \& Related Disabilities
}

Gentile JP. Int J Autism \& Relat Disabil: IJARD-110.

\section{Aggression in Intellectual Disability}

\section{Julie P. Gentile*}

Department of Psychiatry, Wright State University, USA

"Corresponding author: Julie P. Gentile, Department of Psychiatry, Wright State University, USA. Tel: +19372238840; Email: julie.gentile@wright.edu

Citation: Gentile JP (2018) Aggression in Intellectual Disability. Int J Autism \& Relat Disabil: IJARD-110. DOI: 10.29011/IJARD110. 000010

Received Date: 30 July, 2018; Accepted Date: 03 September, 2018; Published Date: 10 September, 2018

\section{Description}

Aggression in its various forms (verbal, physical, property destruction, and auto-aggression or self-injurious behavior) is the most frequent cause for mental health appointments and assessments in patients with Intellectual Disabilities (ID) [1-3]. Patients with ID experience psychiatric and behavioral problems at three to six times the frequency of the general population [4]. The impact of aggression is substantial. For the patient, it can equate to a decrease or termination of family involvement, social isolation, or placement in more restrictive environments. For the caregiver, it can result in negative transference toward the individual with ID, caregiver anxiety, burnout, or injury. For society, it often results in the cost of resources such as frequent hospitalization or incarceration.

Aggression is multi-determined problem and is influenced by biological, psychological, social, spiritual, and cultural factors. Psychiatric and behavioral interventions must be tailored to meet the specific needs of the particular individual with ID and aggression. There rarely is an easy answer to the etiology of aggression; however, use of the biopsychosocial formulation can act as a template upon which to build and with which to perform the detective work necessary.

Rueve and Welton (2008) [3] have posed the question: 'Are aggression and mental illness co-occurring conditions, inter-related, or merely coincidental?' We should ask the same of aggression and ID. Aggression in the ID population often is studied in the context of the entire spectrum of challenging behaviors that can be seen, including stereotyped behavior, pica, rumination, noncompliance, and nonspecific behavior problems [5]. Recent empirical findings about neuroreceptor correlates of aggression can help guide the clinician in selecting appropriate treatments for impulsivity and aggression. Because aggression cannot be understood outside of its situational context, the use of the "biopsychosocial formulation" when evaluating aggression is important. A biopsychosocial formulation includes "predisposing," "perpetuating" and "protective" factors, and is essential in determining the etiology of aggression and in facilitating a proper diagnosis prior to prescribing psychotropic medication. For example, medical conditions should be ruled out through expanded screening laboratory values, a physical examination, and interdisciplinary referrals and consultations when needed.

For patients with ID, "aggression" might be viewed as an externalizing behavior symptom-- a sign --and really should be a stimulus to pursue detective work to identify an etiology. It is important for the clinician to be aware of the most commonly missed, untreated or under-treated medical conditions in persons with ID, as these may present as "behavior problems." The psychiatrist may be the first physician to evaluate a patient with ID because the patient often presents first with "problem behavior" due to an inability to communicate real physical complaints and other medical symptoms. For example, a patient could present with repetitive biting his fist, viewed as "aggression." However, this patient should be evaluated for possible medical conditions of the upper gastrointestinal tract, upper respiratory tract, and the oral cavity, as well as conditions affecting the hands. In addition to possible medical causes, there appear to be time periods when patients with mental health diagnoses exhibit a higher incidence of aggression, including at the time of admission to the hospital as well as the first weeks after discharge from the hospital [3].

Immediately after discharge, it is likely that patients continue to experience active symptoms during this critical period of stabilization. By way of comparison, in the general population, aggression is most frequent in persons with dementia, those who have experienced loss of independence and/or physical functioning, persons suffering from grief and loss, persons who are trying to escape or avoid unwanted demands or situations, and persons who are seeking attention or who are bored [3]. Tenneij et al. (2009) [1] studied aggression in longer-term inpatient treatment centers created for individuals with both mild ID and challenging 
behavior. The vast majority of aggressive incidents was toward direct care staff and precipitated by denial of requests. Among the subjects in Tenneij's study, higher rates of aggression were associated with more severe ID, and in particular Self-Injurious Behavior (SIB) was associated with more severe or profound ID. The two age groups most likely to exhibit aggression were late adolescence and early adulthood; those at highest risk were men aged 20 to 35 years [1]. Deb et al. (2007) and Deb et al. (2008) [6,7] found that higher rates of SIB were associated with more severe ID, poorer communication abilities, and autism. McClintock found aggression was most closely related to visual impairment, hearing impairment, inability to express needs, and inability to ambulate, all of which presumably resulted in unfilled needs $[6,7]$.

Rojahn et al. (2004) [8] studied SIB and aggression and their relationship to impulse control and conduct disorders. Stereotyped behavior was linked more often to intellectual disability and less to schizophrenia. Several other studies have produced consistent findings to support a potential link between SIB/aggressive behavior and affective disorders, and this association also has been suggested by previous authors [8].

Many researchers have studied risk factors for aggression in inpatient treatment facilities for persons with ID [1,9-11]. Behaviors and risk factors which predicted aggression have included history of violence, male gender, ages 20 through 35 years, more severe levels of ID, and presence of personality disorders. The factors more loosely related but thought to play a role included psychiatric pathology, presence of SIB, and antisocial behavior [1]. Aggressive incidents were assessed using the Staff Observation Assessment Scale-Revised or SOAS-R [12]. Previously, Nijman and Palmstierna (2002) [12] had found that at least four types of behaviors were associated with and may be predictive of severe aggression on inpatient units for individuals with ID: 1) antisocial behaviors, 2) impulse control problems, 3) psychotic symptoms, and 4) mood related symptoms (i.e. mood swings, sudden mood changes, and easily upset). Tenneij et al. (2009) [1] also observed that auto-aggression (SIB) may be a unique feature to the ID population over and above the aforementioned factors, which is indicative of severity of aggression, overall, and this has been corroborated by other studies.

Earlier studies have reported widely ranging prevalence rates for SIB in persons with ID, ranging from $1.7 \%$ to $41 \%$ [13]. SIB is a very serious condition for a patient, and a clearer estimate of prevalence rate is necessary. More recent research by Cooper et al. (2009) [13] found the point prevalence of SIB to be approximately $4.9 \%$, and a two-year remission rate of $38.2 \%$ (point prevalence being the proportion of people in a population who have a disease or condition at a particular time or date). Cooper et al. (2009) [13] identified three risk factors in adult patients with ID and SIB that may make SIB more likely to occur: lower cognitive ability, diagnosis of autism, and more serious communication impairments. Other factors which may be associated, but not reaching statistical significance in the Cooper et al. study, included not living with family, not having Down syndrome, diagnosis of attention deficit hyperactivity disorder, visual impairment, and requiring a high level of support in a residential setting. Institutional populations had a higher prevalence of SIB, but this finding was attributed to the increased risk of this subset of the population being admitted to these facilities. No significant association was found between gender and SIB.

Given the unexpectedly high rate of remission of SIB at the two-year mark (38.2\%), [13] concluded that self-injurious behavior may not be as persistent and longstanding as previously thought. The authors also emphasized that the clinician must be cognizant that patients with ID and SIB may have a medical cause and/or suffer medical complications from this behavior, and these must be diagnosed and treated. The most prevalent complications the authors identified were infections and other physical consequences of seclusion and restraint, and the most common medical causes were sensory impairment and side effects of psychotropic medication. Also identified causes and consequences were isolation from supportive persons and other psychological or social consequences resulting from the behavior [13].

\begin{tabular}{|c|}
\hline $\begin{array}{c}\text { Identify genetic syndromes with known behavioral or psychiatric } \\
\text { phenotypes. }\end{array}$ \\
\hline $\begin{array}{c}\text { Establish any psychiatric diagnoses, based on most recent } \\
\text { standardized evidence based diagnostic manual. }\end{array}$ \\
\hline $\begin{array}{c}\text { Determine if there are undetected or untreated medical problems. } \\
\text { in behavior. }\end{array}$ \\
$\begin{array}{c}\text { Consider possible role of drug effects including toxicity/delirium, } \\
\text { side effects (akathisia, disinhibition), withdrawal effects, interaction }\end{array}$ \\
\hline $\begin{array}{c}\text { Identify objective measures of symptoms or behaviors: use screening } \\
\text { tools as appropriate, measurement of behaviors, assessment of } \\
\text { vegetative functions, mood, memory and other mental status } \\
\text { information. }\end{array}$ \\
\hline \begin{tabular}{c} 
Look for any correlation with stressful life events and changes. \\
\hline Assess all environments including structure/supports to meet \\
cognitive developmental needs.
\end{tabular} \\
\hline $\begin{array}{c}\text { Collect detailed data on baseline functioning of the individual. } \\
\text { Assess the individual's abilities to describe internal states and other } \\
\text { communicative abilities. }\end{array}$ \\
\hline $\begin{array}{c}\text { Determine behavioral repertoire, including areas of strength and } \\
\text { weaknesses. }\end{array}$ \\
\hline
\end{tabular}


Assess probable functions of the aggressive behavior, including escape, attention, communication, expression of pain or frustration, modulation of stimulation levels, secure tangibles. Triggers? Factors that maintain behavior? Quantify of frequency and severity. Identify variables contributing to a lowering of the threshold for aggression.

Identify changes in behavioral or other psychosocial treatments correlated with increased problems.

Clarify past treatment trials (What helped? What did not help? Was trial adequate? Were there possible confounding variables? Were successful interventions prematurely terminated?

Table 1: Comprehensive Assessment of People with Intellectual Disabilities and Aggressive Behavior (Charlot and Shedlack).

Table: Conditions to be considered as etiologies of aggression (adapted from Rueve M and Welton R. Violence in Mental Illness. Psychiatry (Edgemont) May 2008 (pages 38-39) and Ryan R. Intensive Conference Dual Diagnosis, Denver, CO. CME Event (July, 2003) and Charlot L, Shedlack K. Masquerade: Uncovering and treating the many causes of aggression in individuals with developmental disabilities [14].

Means of expressing frustration, fear, injustice or anger.

Learned problem behavior.

Relief from boredom.

Modulation of stimulating environments.

Expression of physical pain or acute medical condition.

Means of communication.

Signal of acute psychiatric problem.

Regression in situations of stress, pain, change in routine, or novelty.

\section{Dementia.}

Loss of independence and/or physical functioning.

Grief and loss issues.

Escape or avoidance of unwanted demands or situations.

Attention seeking.

Dementia.

Loss of independence and/or physical functioning.

Grief and loss issues.

Escape or avoidance of unwanted demands or situations.

Attention seeking.

Self-stimulatory behavior.
Product of past experiences.

Parental hostility, maternal permissiveness, absence of maternal affection.

Poor parental modeling limited social supports, poor school experience.

There are several psychiatric diagnoses known to be associated with aggression. These include substance abuse disorders, psychotic disorders (especially those including paranoia), affective or mood disorders, personality disorders (especially antisocial and borderline), conduct disorder, oppositional defiant disorder, sexual sadism, pervasive developmental disorders, and delirium/dementia [3].

There are also many medical conditions that are directly associated with aggression. These include traumatic brain injury (in particular, injuries that are more severe and involve loss of consciousness), intracranial pathology (including tumors, infectious processes, and cerebro-vascular conditions), and metabolic disturbances (including thyroid or other hormonal conditions). Also important to consider are systemic infections, environmental toxins, and certain neurological conditions such as complex partial seizures or temporal lobe foci on abnormal electroencephalograms.

Prefrontal or frontal lobe disorders may play an important role in aggression. The frontal lobe is responsible for executive functioning; this includes such cognitive processes as the ability to recognize consequences resulting from actions. When the patient makes a choice between good and bad, or better and best, this logic and reasoning requires intact frontal lobe functioning [3]. The frontal cortex also likely is the area of the brain associated with the ability to override or suppress unacceptable social responses; frontal lobe function is associated with planning, organizing, and filtering behavioral responses. Because of limitations in frontal lobe functioning, these cognitive processes may be problematic in persons with ID.

Temporal lobe dysfunction also can have a direct effect on the likelihood of aggression. The temporal lobe and its associated structures are involved in fear and response to danger [3]. Patients with ID are likely to have co-occurring neurologic conditions or dysfunction in the temporal lobes. Patients with ID who exhibit aggression and SIB are a heterogeneous group, and their behavior reflects various biologic, psychodynamic, and social factors. There are several extremely rare syndromes known to be associated specifically with SIB in the ID population: Lesch Nyhan Syndrome, Smith Magenis Syndrome, Cornelia deLange Syndrome, and Prader Willi Syndrome [15].

There are several static and dynamic risk factors which have a direct association with aggression both in the general population 
as well as in persons with ID. The static risk factors include past violence (well-documented to have a direct correlation and to be the most reliable indicator), male gender, younger adult age, cognitive deficits, brain injury, dissociative states, military service, weapons training, and major mental illness [3]. The dynamic risk factors include persecutory delusions, command hallucinations, noncompliance, impulsivity, low global assessment of functioning, homicidality, depression, hopelessness, suicidality, and access to weapons [4].

Patients with ID have some additional risk factors for aggression. Aggression in patients with ID can be a means of expressing frustration, a learned problem behavior, an expression of physical pain, an acute medical issue including side effects of medication, a signal of an acute psychiatric problem, or regression in situations of stress, pain, change in routine, or novelty $[3,5,6]$. SIB that is associated with severe/profound ID is not uncommon, and other disabilities, especially cerebral palsy and sensory impairment, can further complicate the assessment of SIB in this population $[5,6]$. There also is an association between sleep problems and daytime challenging behavior (irritability, SIB, hyperactivity, and screaming) [16,17]. Aggression in children with ID is associated with co-occurring sleep problems, more severe ID, poly-pharmacy, seizure disorders, and cerebral palsy [16,17].

Aggression toward others is more likely to be exhibited by persons with less severe cognitive deficits with greater verbal communication skills, while SIB is more common in individuals with severe ID, who may have decreased mobility, reduced selfhelp skills, more severe hearing impairment, increased stereotypic movements, and less well developed communication skills.

Transitions are notoriously difficult for individuals with ID and they experience many of them. The change from an educational setting to occupational setting may be associated with new residential placement as well, for example. Introduction to school means introduction to a wide variety of new experiences, more noise, transitions, and other factors that can precipitate SIB or other aggression. This also may be a transition time for peers, siblings, parents, and others, and therefore makes this a particularly vulnerable time for resurfacing of grief and loss issues. Family members themselves may experience symptoms of grief and loss as the individual with ID goes through various developmental stages, or when these stages would normally have appeared in a child or sibling without ID (entering school, graduating high school, attending college, marriage and having children). Irrespective of the person's communication skill level, if aggression continues to be a more powerful means of gaining attention than other behaviors that have been tried, then treatment is not likely to be effective [18].

In this scenario, patients with ID often begin to use violence to respond to or communicate about psychosocial stressors, as their cognitive deficits make it difficult for them to develop more adaptive, nonviolent ways of responding $[3,7,19]$. They need to be helped to learn new ways, and to experience less reinforcement (reward) for using aggression, in order to change.

In proposing the biopsychosocial model of disease in 1977, George Engel said: "(in order) to provide a basis for understanding the determinants of disease and arriving at rational treatments and patterns of health care, a medical model must take into account the patient, the social context in which he lives, and the complementary system devised by society to deal with the disruptive effects of the illness" [20]. The biopsychosocial model is useful when attempting to determine the etiology of aggression in a person with ID. The model or formulation involves gathering information through a variety of sources: patient interview, discussion with family members and/or caretakers, review of clinical records, and contact with collaborating agencies. This leads to a formulation of the problem(s), diagnosis and treatment plan [20]. The biopsychosocial model allows for an assessment that evolves over time and which should be updated serially, even for patients well-known to the physician. Some of its components can vary from assessment to assessment or even from day to day depending on the disorder and the "supporting, predisposing, perpetuating, perpetual, and protective factors" related to the disorder.

When evaluating a patient with ID who has shown aggression periodically over time, the clinician must ask about onset and chronology of the aggression and all other associated symptoms. It is important to elicit not only the presenting problem, but determine who has defined the problem, how long it has been observed, in what environments it is observed and, if long-standing in nature, why evaluation is being sought at this point in time. It is crucial that the person making the referral for evaluation is present to explain particular concerns. The patient's pre-morbid functioning must be understood and described in detail. If a symptom waxes and wanes in intensity or varies with level of stress or in different environments, any pertinent details regarding these circumstances must be documented. If a behavior symptom only occurs at home or only in the occupational setting, this is important information. If the behavior increases in frequency or intensity at certain times, or only exists at a certain time of day, a specific environment, before or after certain events (i.e. family visits), or when a certain staff member or family member is present, this can narrow down the differential diagnoses. The behavior may signal a problem for the person that is interpersonal, physical, or environmental. The clinician must also take into consideration various mental health issues as a possible etiology of the behavior. When was this individual last doing well? What did that look like (i.e. what is their baseline)? Is the care that is provided consistent across shifts and environments? Is there a conflicted relationship with family or other important people? Is there consistent medication administration? Extract every detail about the time period(s) 
surrounding the incident(s) of aggression, as they can paint a picture that will help in assessing whether treatment is effective.

The development of a differential diagnosis of aggression can lead to possible treatments that identify target behaviors that may be responsive to medication, such as a mood disorder that is manifesting itself by aggressive behavior associated with a sleep disturbance. Once this is accomplished, it is important to establish the baseline rate of target behaviors in order to monitor response to treatment. A behavioral psychologist or behavior support specialist can help design, monitor, and educate staff/direct caregivers regarding data collection. It is a challenge to develop broad and all-inclusive treatment recommendations because of the heterogeneity of aggression. Treatment choices, including the use of medication, should be guided by the biopsychosocial model, with ongoing assessment of the total picture. There are multiple variables to consider when using medications in the ID population, and sometimes they are not indicated. For example, to quote [21]. "Antipsychotics are the most widely prescribed medications in individuals with intellectual disability even if schizophrenia and other psychotic disorders do not affect more than 3\% of such population" [21]. Antipsychotic medications have in the past sometimes been inappropriately prescribed for patients with aggression for their tranquilizing effects alone. However, self-report of side effects is often compromised in patients with ID given their communication limitations. It is estimated that approximately $20 \%$ of patients with ID experience adverse drug reactions, with some side effects manifesting more severely [22]. Neuroleptic malignant syndrome is more frequently fatal in patients with ID at rates twice that of the general population [22]. There is evidence that the ID population likely experiences increased rates of sensitivities and adverse drug reactions with psychotropic medication probably as a consequence of damage to the central nervous system, and also metabolic, pharmacodynamic and pharmacokinetic differences [23]. Therefore, all psychotropic medications must be used with special caution.

Appropriate management, when psychopharmacologic drugs are needed, includes smaller starting doses for children and adolescents than their normally developing peers, and these doses should be increased at slower rates. Treatment plans should not be based on pharmacology alone. Skill deficits, unmet sensory needs and frustration because of absence of meaningful choices should be addressed.

Expert consensus guidelines recommend that treatment should be based upon the most specific psychiatric diagnosis possible $[6,13,19]$. When only a tentative, nonspecific diagnosis can be made, as in the presence of severe/profound ID, clinicians should focus on one or more behavioral symptoms as targets of treatment. Psychotropic medication may or may not be one component of treatment, but it should not be the only treatment strategy.
In general, treatment with pharmacologic agents for the patient with ID does not differ much from pharmacotherapy in patients without ID. Risperidone and aripiprazole are well documented to be effective in the treatment of children with ID, particularly those with autism. It is the preferred agent in the treatment of disruptive behavior in children and adolescents with ID $[22,24]$. The selection of treatment agents for disruptive behavior in patients with ID depends on the severity of symptoms and the extent to which social, biological, or psychological factors cause the symptoms. Disruptive behavior needs to be addressed (whether with pharmacotherapy or alternate interventions) despite the fact that there are neither clear diagnostic criteria nor consistent ways to measure it. Medications have been shown to help decrease impulsivity, aggression, and agitation regardless of their etiology. Patients with ID in general have a high prevalence of disinhibition, impulsive behavior and a lack of the 'filtering' in addition to differing degrees of executive function deficits compared to the general population. Pharmacotherapy is one of the basic options for the treatment of challenging behavior after several requirements are met. Informed consent issues must be carefully attended to and special consideration utilized with patients with cognitive deficits who are acting as their own guardians. Physical and psychological conditions must be evaluated, diagnosed and appropriately treated. Any medication decision must involve careful consideration of risks and benefits. Reviews of the many of the expert consensus guidelines instruct the prescriber to start low and go slow. A number of side effects can be more severe or have variant presentations compared to similar treatment in patients without ID, and patients may not be able to verbally report them. Use of standardized and universal rating scales should be used to identify and track targets symptoms, extrapyramidal symptoms and to screen for metabolic side effects. In case of no response to monotherapy, combinations of psychotropic medications may be useful, but attention to and prevention of unnecessary polypharmacy must be avoided. Diagnosing and treating aggression in a patient with ID requires careful detective work. The mainstay of the detective work lies in utilizing a comprehensive biopsychosocial formulation, and taking the time to determine the most appropriate interventions and treatment measures. This includes strategic use of collateral data from all environments, and from various instrumental people in the patient's life.

\section{Summary}

In summary, utilization of the biopsychosocial formulation and incorporation of the developmental stage where the patient lives will allow the clinician to accurately diagnose and treat patients with ID when they present with aggression. Medications should be used in combination with many other interventions and ancillary services. If the aggression or SIB can be defined in terms of a target symptom or behavior, a behavior support specialist can 
suggest ways to collect quantitative and qualitative data including the frequency, severity and associated environmental variables.

\section{References}

1. Tenneij NH, Didden R, Stolker JJ, Koot HM (2009) Markers for aggression in inpatient treatment facilities for adults with mild to borderline intellectual disability. Research in Developmental Disabilities 30: 1248-1257.

2. Hurley AD, Levitas A, Lecavalier L, Pary RJ (2007) Assessment and Diagnostic Procedures. In Fletcher et al. eds (2007). Diagnostic Manual -- Intellectual Disability (DM-ID): A Textbook of Diagnosis of Mental Disorders in Persons with Intellectual Disability. Kingston, NY: NADD Press.

3. Rueve M, Welton R (2008) Violence in Mental Illness. Psychiatry (Edgemont) 5: 35-48.

4. Larson SA, Lakin KC, Anderson L, Kwak N, Lee JH, et al. (2001) Prevalence of mental retardation and developmental disabilities: Estimates from the 1994/1995 National Health Interview Survey Disability Supplements. Am J Ment Retard 106: 231-252.

5. Antonnacci DJ, Manuel C, Davis E (2008) Diagnosis and treatment of aggression in individuals with developmental disabilities. Psychiatr $Q$ 79: 225-247.

6. Deb S, Unwin GL (2007) Psychotropic medication for behaviour problems in people with intellectual disability: A review of current literature. Current Opinion in Psychiatry 20: 461-466.

7. Deb S, Chaplin R, Sohanpal G, Unwin G, Soni R, et al. (2008) The effectiveness of mood stabilizers and antiepileptic medication for the management of behavioural problems in adults with intellectual disability: a systematic review. Journal of Intellectual Disability Research 52: $107-113$.

8. Rojahn J, Matson JL, Naglieri JA, Erik Mayville (2004) Relationships between psychiatric conditions and behavior problems among adults with mental retardation. American Journal on Mental Retardation 109: 21-23.

9. McClintock K, Hall S, Oliver C (2003) Risk markers associated with challenging behavrios in people with intellectual disabilities: A metaanalytic study. Journal of Intellectual Disability Research 47: 405-416.

10. Tyrer F, McGrother C, Thorp C, Donaldson M, Bhaumik S, et al. (2006) Physical aggression towards others in adults with learning disabilities: Prevalence and associated factors. Journal of Intectual Disability Research 50: 295-304.

11. Hogue T, Steptoe L, Taylor J, Lindsay W, Mooney P, et al. (2006) A comparison of offenders with intellectual disability across three levels of security. Criminal Behavior and Mental Heatlh 16: 13-28.
12. Nijman $\mathrm{H}$, Palmstierna $\mathrm{T}$ (2002) Measuring aggression with the staff observation aggression scale-revised. Acta-Psychiatrica Scandinovvica Supplement 412: 101-102.

13. Cooper S-A, Smiley E, Allan LM, Jackson A, Finlayson J, et al. (2009) Adults with intellectual disabilities: prevalence, incidence and remission of self-injurious behaviour, and related factors. J of Intellec Disabil Res Special Issue 53: 200-216.

14. Charlot L, Shedlack K (2002) Masquerade: Uncovering and treating the many causes of aggression in individuals with developmental disabilities. NADD Bulletin 5: 59-64.

15. Fletcher R, Loschen E, Stavrakaki, C, First M (2007) Diagnostic Manual -- Intellectual Disability (DM-ID): A Textbook of Diagnosis of Mental Disorders in Persons with Intellectual Disability.

16. Didden $R$, Korzilius $H$, van Aperlo $B$, van Overloop $C$, de Vries $M$ (2002) Sleep problems and daytime problem behaviours in children with intellectual disability. J Intellect Disabil Res 46: 537-547.

17. Allen DG, Lowe K, Moore K, Brophy S (2007) Predictors, costs and characteristics of out of area placement for people with intellectual disability and challenging behaviour. J Intellect Disabil Res 51: 409-416.

18. Matson JL, LoVullo SV (2008) A Review of Behavioral Treatments for Self-injurious Behaviors of Persons with Autism Spectrum Disorders. Behav Modif 32: 61.

19. Aman MG, Crismon ML, Frances A, King BH, Rojahn J, eds., 2004 Treatment of psychiatric and behavioral problems in individuals with mental retardation: An update of the expert consensus guidelines for mental retardation/developmental disability populations. Englewood, CO: Postgraduate Institute for Medicine.

20. Campbell WH and Rohrbaugh RM. The Biopsychosocial Formulation: A guide for mental health professionals. New York, Routledge, 2007.

21. La Malfa G, Lassi S, Bertelli M, Castellani A (2006) Reviewing the use of antipsychotic drugs in people with intellectual disability. Hum Psychopharmacol Clin Exp 21: 73-89.

22. Habler F, Reis O (2010) Pharmacotherapy of Disruptive Behavior in Mentally Retarded Subjects: A Review of the Current Literature. Developmental Disabilities Research Reviews 16: 265-272.

23. Janowsky DS, Shetty M, Barnhill LJ (2005) Serotonergic antidepressant effects on aggressive, self-injurious and destructive/disruptive behaviors in intellectually disabled adults: a retrospective, open-label, naturalistic trial. International Journal of Neuropsychopharmacology 8: 37-48.

24. Volavka J, Citrome L, Huertas D (2006) Update on the biological treatment of aggression. A Review. Actos Esp Psiquiatr 34:123-135. 